\title{
The Prevalence of Asthenopia and its Determinants Among Schoolchildren
}

\author{
Hassan Hashemi, ${ }^{1}$ Mehdi Khabazkhoob, ${ }^{2,3}$ Samira Forouzesh, ${ }^{4}$ Payam Nabovati, ${ }^{3}$ Abbas Ali Yekta,,${ }^{4}$ \\ and Hadi Ostadimoghaddam ${ }^{5}$ \\ ${ }^{1}$ Noor Research Center for Ophthalmic Epidemiology, Noor Eye Hospital, Tehran, Iran \\ ${ }^{2}$ Department of Medical Surgical Nursing, School of Nursing and Midwifery, Shahid Beheshti University of Medical Sciences, Tehran, Iran \\ ${ }^{3}$ Noor Ophthalmology Research Center, Noor Eye Hospital, Tehran, Iran \\ ${ }^{4}$ Department of Optometry, School of Paramedical Sciences, Mashhad University of Medical Sciences, Mashhad, Iran \\ ${ }^{5}$ Refractive Errors Research Center, School of Paramedical Sciences, Mashhad University of Medical Sciences, Mashhad, Iran \\ "Corresponding author: Abbas Ali Yekta, Department of Optometry, School of Paramedical Sciences, Mashhad University of Medical Sciences, Mashhad, Iran. E-mail: \\ yektaa@mums.ac.ir
}

Received 2016 October 19; Revised 2016 December 25; Accepted 2017 January 21.

\begin{abstract}
Objectives: The current study aimed at determining asthenopia prevalence and its associated factors in a population of high school students.

Methods: In the current cross sectional study, samples were selected from high school students (range, 12 to 18 years) in Kermanshah city, Iran, through stratified cluster sampling. Any person with at least 1 symptom was considered to have asthenopia.

Results: Of the 1070 selected samples, 1040 participated, and examinations were completed for 901 students. The prevalence of asthenopia was $49.4 \%$ ( 45.7 to 53.2). The prevalence of asthenopia was 62.8\% (51.9 to 73.8) and 47.7\% (43.8 to 51.7) in males and females, respectively $(\mathrm{P}=0.013)$. Asthenopia prevalence increased from $21.4 \%$ in 12 -year-old to $63.9 \%$ in 18 -year-old cases $(\mathrm{P}<0.001)$. The most common symptom in the study was tearing (20.03\%) and eye pain (19.88\%) during near-work and reading.

Conclusions: The prevalence of asthenopia in this student population was high. Since asthenopia can interfere with near-work, its treatment merits high importance in school-age children.
\end{abstract}

Keywords: Asthenopia, Cross Sectional Study, Schoolchildren, Middle-East

\section{Background}

Asthenopia is a term used to describe a variety of nonspecific symptoms associated with the visual system, especially during nea-work (1). Asthenopia is examined subjectively. This subjective problem entails 2 groups of symptoms; nonspecific symptoms including fatigue, burning, irritation, eye pain, aching eyes, sore eyes, and headache, and specific symptoms are defined as symptoms such as photophobia, blurred vision, double vision, itching, tearing, dryness feeling, and foreign body sensation (2).

Previous studies indicate that asthenopia mostly occurs due to uncorrected refractive errors, (3) accommodative dysfunctions $(4,5)$, vergence anomalies $(6,7)$, inappropriate lighting condition (8), extraocular muscle imbalance (9), and compromised quality of the viewed image such as poor contrast (10). Thus, asthenopia is categorized accordingly as internal and external. Internal asthenopia is caused by problems such as uncorrected refractive errors and accommodation disorders, while insufficient ambient lighting is responsible for external asthenopia (2). This eye problem interferes with near-work, especially in children and can interfere with attention and academic performance (11).

Along with increased use of computer devices and other near-work in children, the prevalence of asthenopia and its associated complications are expected to increase (12). Nonetheless, authors' knowledge about the prevalence of this subjective problem is limited to few studies. Review of different studies in children show that the prevalence of asthenopia and certain symptoms range from below $15 \%$ to over $80 \%$, and headaches are 1of the most common symptoms reported in most studies (13). Although many studies are conducted on computer operators to show the prevalence of asthenopia, $(1,14)$ few reports are available regarding schoolchildren $(11,15)$. Moreover, studying the causes of asthenopia can improve the ability to treat this condition and resolve symptoms in affected individuals. In Iran, some studies assessed vision problems in schoolchildren (16-18); however, asthenopia is not studied in any age group of Iranian students. The current study was conducted to examine the prevalence of asthenopia and its related factors in a student population in Iran.

Copyright (c) 2017, Iranian Society of Pediatrics. This is an open-access article distributed under the terms of the Creative Commons Attribution-NonCommercial 4.0 International License (http://creativecommons.org/licenses/by-nc/4.0/) which permits copy and redistribute the material just in noncommercial usages, provided the original work is properly cited. 


\section{Methods}

The current cross sectional study was conducted in 2015 in Kermanshah, a city in the West of Iran. The target population was high school students in Kermanshah. Samples were selected through multi-stage cluster sampling. First, a number of high school male and female students were selected. Then, from each school, a number of students in each class were randomly selected proportional to the total number of students in the school. After sample selection, consent forms were distributed to be signed by parents.

\subsection{Examination}

On the examination day, having a signed consent form was the first inclusion criteria. Before the examinations, each student was interviewed and asked a number of questions regarding his/her near-work and studying habits. Upon entering the exam room, students were first tested 3 times for non-cycloplegic auto-refraction by a skilled operator using the TOPCON RM 8800 (Topcon Corporation, Tokyo, Japan); the results were recorded, and printouts were attached to their files. In the next stage, all participants were tested for uncorrected visual acuity (UCVA). Then, auto-refraction results were refined through retinoscopy, using HEINE BETA 200 (HEINE Optotechnik, Germany) and the MSD trial lenses (MSD Meniscus Trial Lenses, Italy). For each student, first the right and, then, the left eye was tested. For any case with UCVA worse than 20/25 in either eye, subjective refraction was done and the best corrected visual acuity (BCVA) was recorded. Then, far (6 meters) and near $(40 \mathrm{~cm})$ cover tests were done using accommodative targets (a single letter 1 line above acuity threshold) and the alternate cover test with prism bar was carried out to diagnose and measure phoria. In the next stage, the near point of accommodation (NPA) was measured with the Donder push-up method using Royal Air Force Rule (RAF) and near print equivalent to 20/25 VA as accommodative target. The target was slowly moved closer to the child along the midline and he/she was asked to report the first sustained blur. To increase the reliability of the test, the measurement was done 3 times and the average NPA was recorded. The average NPA was, then, converted to amplitude of accommodation (AA) in Diopter. The near point of convergence (NPC) was measured similar to AA; instead, the participant was instructed to report the first sustained diplopia of the target. Again, the NPC was measured 3 times and the average was recorded.

The gradient accommodative-convergence / accommodation (AC/A) ratio was determined by re-measurement of near phoria by adding -1.00 minus lenses to subjective refraction and comparing the result with the baseline near phoria value. All optometric tests were completed by an experienced optometrist.

\subsection{Definition of Asthenopia}

To diagnose asthenopia, the 10 symptoms of eye pain, dry eyes, eye swelling, blurred vision, diplopia, foreign body sensation, photophobia, tearing, decreased visual acuity, and difficulty in sustaining visual operations were investigated in detail through face to face interviews by a trained interviewer. In line with previous studies $(1,15)$, any person with at least 1of these symptoms was considered to have asthenopia. Nonetheless, to show its degree, the percentage of people with at least 2, 3, and 4 symptoms was also determined.

Exclusion criteria included BCVA of less than 20/25 in either eye, strabismus, ocular or systemic diseases affecting binocular vision, and using any medication that can impact accommodation or convergence.

\subsection{Statistical Analysis}

The prevalence of asthenopia was presented in percentage along with 95\% confidence intervals (CI). Logistic regression was used to examine possible relationships, and mean values of quantitative variables were compared between the cases with and without asthenopia using the $t$ test. A significance level of 0.05 was applied in all statistical tests.

\subsection{Ethical Issues}

The ethics committee of Mashhad University of Medical Sciences approved the study protocol conducted in accordance with the tenets of the Helsinki Declaration. All participants' parents signed a written informed consent.

\section{Results}

Of the 1070 selected students, 1040 were included in the study; the exclusion criteria were applied, and the results of 901 students are presented; their mean age was 15.1 \pm 1.6 years.

Mean spherical equivalent refraction in the total sample was $-0.96 \pm 1.13 \mathrm{D}$; it was $-0.96 \pm 1.18 \mathrm{D}$ and $-0.97 \pm 1.13 \mathrm{D}$ in females and males, respectively $(\mathrm{P}=0.964)$.

The prevalence of asthenopia by age, gender, and degree of asthenopia is summarized in Table 1. Based on the definition of having at least 1symptom, 49.4\% (45.7 to 53.2) of the students had asthenopia, and the prevalence of having at least 2, 3, and 4 symptoms was $24.9 \%$ (21.7 to 28.2 ), $14.4 \%$ (11.8 to 17.0 ), and 9.1\% (6.9 to 11.2 ), respectively. The prevalence of asthenopia was $62.8 \%$ (51.9 to 73.8 ) in males and $47.7 \%$ (43.8 to 51.7) in females $(P=0.013$, odds 
ratio $=1.85$, 95\% CI: $1.14-3.0)$. Also, as presented in Table 1 , the prevalence increased from $21.4 \%$ in 12-year-old cases to $63.9 \%$ in 18 -year-old cases $(\mathrm{P}<0.001)$. Asthenopia with at least 4 symptoms was not observed in 12-year-old cases, while $19.4 \%$ of the 18-year-old age group had at least 4 symptoms. As illustrated in Figure 1, the most common symptoms were tearing and eye pain during near-work and reading, while difficulty in sustaining visual operations was reported by only $0.14 \%$ of the students. As presented in Table 2 , the average time spent using cell phones and computers was significantly higher in students with asthenopia, while most of the other activities were not significantly different between the 2 groups. Table 3 summarizes the mean and standard deviation of the AA, NPC, and AC/A ratio in students with and without asthenopia; $t$ test results indicated significantly lower AA and AC/A ratio, and a significantly higher NPC in students with asthenopia. About $50.2 \%$ of the students with normal stereopsis and $47.9 \%$ of those with abnormal stereopsis ( $>120 \mathrm{sec}$ arc) had asthenopia $(\mathrm{P}=0.562)$. Mean near esophoria in students with and without asthenopia was 0.61 and 0.40 prism diopter (PD) $(\mathrm{P}=0.165)$, respectively. The prevalence of significant near exophoria defined with a cutoff point of 6 PD was $54.9 \%$ and $48.3 \%$ in students with and without asthenopia, respectively $(\mathrm{P}=0.142)$.

\section{Discussion}

The current study was the first to describe the prevalence of asthenopia in a population of Iranian students. In this section, the comparison of results with other studies should be performed with caution. One of the reasons for the differences among studies is various definitions of asthenopia that should be considered. As already demonstrated, about $50 \%$ of the students in the study had asthenopia, and 9.1\% reported having 4 symptoms or more. Based on the criteria employed in the current study, Han et al. (15) showed that the prevalence of asthenopia in Chinese students was $57 \%$. Vilela et al. (11) recently reported a prevalence of $24.7 \%$ in children aged 6 to 16 years. According to a recent review, the prevalence of asthenopia in Australian, Swedish, and Indian schoolchildren ranges from $12.6 \%$ to $32.2 \%$ (13). There are studies that have only examined the prevalence of eye strain, and, thus, their results are not comparable to those of the asthenopia studies. In addition to definition differences, age group seems to be another important factor affecting the prevalence of asthenopia. Overall, asthenopia prevalence was not low in the current study. This could be due to heavy studying in many high school students for their pre-college exams and overusing computer devices; although race and ethnic variations should also be considered.
In the current study, a higher percentage of males reported asthenopia symptoms. Ma L (19) showed a higher prevalence in females; while, Han et al. showed no difference between the genders (15). Contradictory results regarding gender-related prevalence rates do not allow reaching a strong hypothesis. However, as already demonstrated, the prevalence of asthenopia significantly increases with aging. This trend was observed even in severe cases of asthenopia. Few studies have focused on how asthenopia changes with age, but overall results indicate that the prevalence is low in childhood. Ip et al. reported a prevalence of $15.2 \%$ in 6-year-old children (20). Sterner found no signs of asthenopia in children under 7.5 years old, while $47.2 \%$ of 7.5 - to 10 -year-old children had at least 1 symptom during near-work (21) Bhandari reported an increase in asthenopia prevalence with age, even in the elderly (1). Mocci also showed changes in asthenopia with age increase (22) It is a little difficult to interpret this finding; 1 important reason for the low prevalence in children is their understanding of the questions, and the answers being subjective. The higher prevalence of asthenopia with increasing age could be because people are more involved in near-work, and in the presence of accommodative and binocular disorders, which are considered as important factors in developing asthenopia (23), the symptom occurs more frequently during near-work. The time spent using computer and cell phone was significantly higher in students with asthenopia. This had been already shown in previous studies, and the main reason seems to be overstimulation of accommodation, imbalance in the convergence and accommodation relationship, and dry eye (21, 24).

As observed in the results, the most common symptom in the current study was tearing followed by eye pain; while, difficulty in sustaining visual operations was the rarest symptom. In the study by Wajuihian (2), headaches were the most common symptoms of asthenopia followed by itching and tearing; while, the least common symptom was diplopia. Mvtu and Kimbo (25), Neugebauer (26), and Alexander et al. (27) also found headaches as the most common symptom of asthenopia; while, Dwyer (2) and McKay (28), and Westman (23) reported near blur or eye strain and tired eyes as the most common symptoms.

The results of the current study showed that the amount of NPC was significantly higher in the group with asthenopia than the normal group; on the other hand, the AC/A ratio was lower in the asthenopia group than the normal group. A remote NPC with a low AC/A ratio suggests a higher prevalence of convergence insufficiency (CI) in the asthenopic cases. $\mathrm{CI}$ is a common binocular vision anomaly associated with high decompensated near exophoria, distance NPC, and a low AC/A ratio; it is sug- 


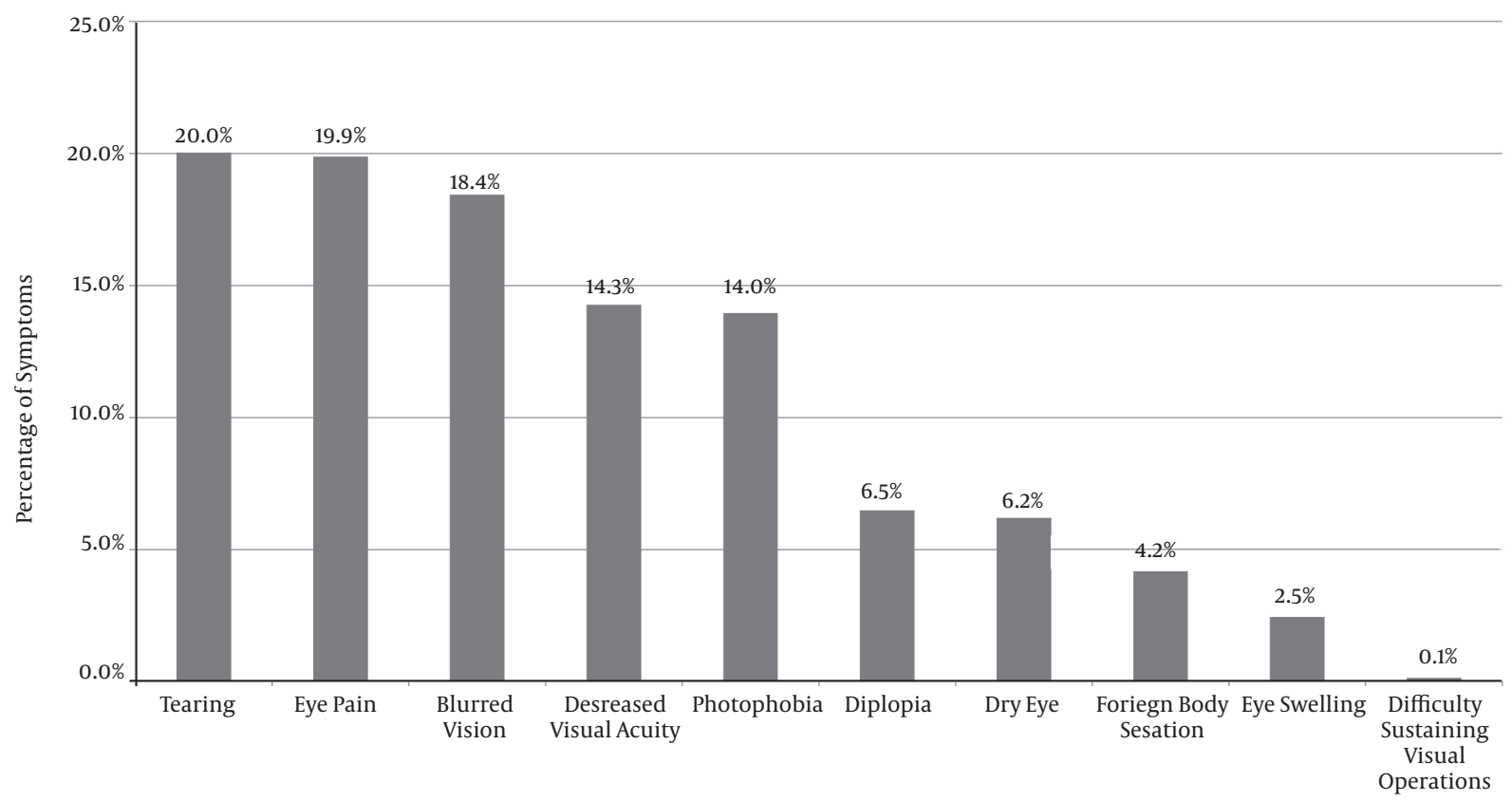

Figure 1. The Prevalence of Symptoms in Children

Table 1. The Prevalence of Asthenopia Among Schoolchildren by Age and Gender ${ }^{a}$

\begin{tabular}{|c|c|c|c|c|}
\hline \multirow[t]{2}{*}{ Variables } & \multicolumn{4}{|c|}{ Number of Symptoms } \\
\hline & 1 or More & 2 or More & 3 or More & 4 or More \\
\hline Total & $49.4(45.7-53.2)$ & $24.9(21.7-28.2)$ & $14.4(11.8-17.0)$ & $9.1(6.9-11.2)$ \\
\hline \multicolumn{5}{|l|}{ Gender } \\
\hline Female & $47.7(43.8-51.7)$ & $24.7(21.3-28.1)$ & $14.1(11.4-16.9)$ & $8.8(6.5-11.0)$ \\
\hline Male & $62.8(51.9-73.8)$ & $26.9(16.9-37.0)$ & $16.7(8.2-25.1)$ & $11.5(4.3-18.8)$ \\
\hline \multicolumn{5}{|l|}{ Age, $y$} \\
\hline 12 & $21.4(10.3-32.5)$ & $5.4(0.7-11.4)$ & 0 & 0 \\
\hline 13 & $37.1(24.7-49.5)$ & $11.3(3.2-19.4)$ & $3.2(1.1-7.7)$ & $1.6(1.2-4.8)$ \\
\hline 14 & $39.3(29.8-48.7)$ & $15.9(8.8-22.9)$ & $8.4(3.1-13.8)$ & $3.7(0.1-7.4)$ \\
\hline 15 & $54.7(47.1-62.3)$ & $28.2(21.4-35.1)$ & $20.6(14.4-26.7)$ & $11.8(6.9-16.7)$ \\
\hline 16 & $61.6(54.1-69.1)$ & $33.5(26.2-40.8)$ & $17.1(11.3-22.9)$ & $12.2(7.1-17.3)$ \\
\hline 17 & $49.5(39.5-59.5)$ & $29.3(20.2-38.4)$ & $17.2(9.6-24.7)$ & $11.1(4.8-17.4)$ \\
\hline 18 & $63.9(47.4-80.4)$ & $38.9(22.2-55.6)$ & $25.0(10.1-39.9)$ & $19.4(5.9-33.0)$ \\
\hline
\end{tabular}

${ }^{\mathrm{a}}$ Data were expressed based on percentage and $95 \%$ confidence interval.

gested as 1 of the most common causes of asthenopic symptoms in children (29). Since up to $6 \mathrm{PD}$ of near exophoria is considered normal and physiological (30), this threshold was employed to categorize near exophoria into 2 groups of high exophoria ( $>6 \mathrm{PD}$ ) and low exophoria ( $\geq 6 \mathrm{PD}$ ). Based on the results of the current study, although the prevalence of high exophoria was higher in the asthenopia group than the normal group, this difference was not statistically significant, which rejects $\mathrm{CI}$ as the primary cause of asthenopic complaints. Another factor that could be attributed to asthenopic complaints is the convergence excess anomaly, but this hypothesis was also rejected as there 
Table 2. Mean and Standard Deviation of Time Spent on Performing Certain Near-Work Activities, Sleeping, and Exercising Among Schoolchildren with and Without Asthenia ${ }^{a}$

\begin{tabular}{lcc}
\hline Variables & Normal & Psthenopia \\
\hline Using computers hour/day, hour/day & $1.29 \pm 1.52$ & $1.58 \pm 1.98$ \\
\hline Using cell phones, hour/day & $2.18 \pm 2.47$ & $2.87 \pm 2.85$ \\
\hline Studying and reading, hour/day & $3.83 \pm 2.25$ & $3.88 \pm 2.22$ \\
Watching TV, hour/day & $2.43 \pm 1.95$ & $2.66 \pm 1.92$ \\
\hline Looking at the board in class, hour/day & $4.99 \pm 1.76$ & 0.001 \\
\hline Sleeping, hour/day & $7.66 \pm 1.72$ & 0.122 \\
\hline Participating in sports and exercise, hour/week & $2.57 \pm 3.53$ & $7.6 \pm 2.05$ \\
\hline
\end{tabular}

${ }^{\mathrm{a}}$ Value are expressed as Mean $\pm \mathrm{SD}$.

Table 3. Mean and Standard Deviation of Amplitude of Accommodation, Near Point of Convergence, and the Accommodative-Convergence Over Accommodation Ratio Among Schoolchildren with and Without Asthenopia ${ }^{a}$

\begin{tabular}{lccc}
\hline Variables & Normal & Asthenopia & P Value \\
\hline AA, diopters & $10.51 \pm 1.87$ & $10.09 \pm 2.22$ & 0.006 \\
NPC, cm & $5.65 \pm 1.81$ & $6.13 \pm 2.36$ & 0.003 \\
AC/A & $2.53 \pm 1.35$ & $2.32 \pm 1.27$ & 0.034 \\
\hline
\end{tabular}

${ }^{\mathrm{a}}$ Value are expressed as Mean $\pm \mathrm{SD}$.

was no significant difference in near esophoria between the 2 groups and the $\mathrm{AC} / \mathrm{A}$ ratio was low. As for AA, it was significantly lower in the asthenopia group, which means a higher prevalence of accommodative insufficiency in this group, and suggests this accommodative anomaly as a potential factor to develop asthenopic complaints. On the other hand, reduced AA can lead to reduced accommodative response and accommodative convergence. This can exert more strain on positive fusional convergence, increased near exophoria, and a more distant NPC due to a decrease in the gross convergence (30), and this explains the higher prevalence of exophoria and higher NPC in the asthenopia group in the current study, compared to the normal group. The current study results were consistent with the findings of Marran et al. (31) and suggest accommodation insufficiency as a major factor responsible for asthenopic complaints in children. Therefore, it is important to diagnose and treat this condition to improve the accommodative performance in children and reduce their asthenopic symptoms.

The current study had some limitations; for example, dry eye objective tests were not applied. Moreover, conducting this study in the Kurdish ethnic group hinders generalizing its results to other Iranian high school students. Therefore, more extensive studies are required in this regard in Iran.

\section{Acknowledgments}

This research was supported by Mashhad University of Medical Sciences, Mashhad, Iran (grant no: 930743).

\section{Footnote}

Financial Disclosure: The authors declared no conflict of interest exists.

\section{References}

1. Bhanderi DJ, Choudhary S, Doshi VG. A community-based study of asthenopia in computer operators. Indian JOphthalmol. 2008;56(1):51-5. doi: 10.4103/0301-4738.37596. [PubMed: 18158404].

2. Wajuihian SO. Frequency of asthenopia and its association with refractive errors. African Vis Eye Health. 2015;74(1):74-80. doi: 10.4102/aveh.v74i1.293.

3. Wiggins NP, Daum KM. Visual discomfort and astigmatic refractive errors in VDT use. J Am Optom Assoc. 1991;62(9):680-4. [PubMed: 1815002].

4. Hennessey D, Iosue RA, Rouse MW. Relation of symptoms to accommodative infacility of school-aged children. Optom Vis Sci. 1984;61(3):177-83. doi: 10.1097/00006324-198403000-00005.

5. Levine S, Ciuffreda KJ, Selenow A, Flax N. Clinical assessment of accommodative facility in symptomatic and asymptomatic individuals. JAm Optom Assoc. 1985;56(4):286-90. [PubMed: 3989210].

6. Sheedy JE, Saladin J. Association of symptoms with measures of oculomotor deficiencies. Optom Vis Sci. 1978;55(10):670-6. doi: 10.1097/00006324-197810000-00002.

7. Scheiman M, Cotter S, Kulp MT, Mitchell GL, Cooper J, Gallaway M, et al. Treatment of accommodative dysfunction in children: results from a randomized clinical trial. Optom Vis Sci. 2011;88(11):1343-52. doi: 10.1097/OPX.0b013e31822f4d7c. [PubMed: 21873922].

8. Committee IOL. . American national standard practice for office lighting. New York: Illuminating Engineering Soci ety of North America; 1982. ANSI/IES RP-1.

9. Sheedy JE, Saladin JJ. Phoria, vergence, and fixation disparity in oculomotor problems printed in U.S.A. Optom Vis Sci. 1977;54(7):474-8. doi: 10.1097/00006324-197707000-00008.

10. Sheedy JE, McCarthy M. Reading performance and visual comfort with scale to grey compared with black-and-white scanned print. Displays. 1994;15(1):27-30. doi: 10.1016/0141-9382(94)90040-x. 
11. Vilela MA, Castagno VD, Meucci RD, Fassa AG. Asthenopia in schoolchildren. Clin Ophthalmol. 2015;9:1595-603. doi: 10.2147/OPTH.S84976. [PubMed: 26357460].

12. Abdi S. Asthenopia in schoolchildren. Institutionen för klinisk neurovetenskap/Department of Clinical Neuroscience; 2007.

13. Vilela MA, Pellanda LC, Fassa AG, Castagno VD. Prevalence of asthenopia in children: a systematic review with meta-analysis. I Pedi$\operatorname{atr}$ (Rio J). 2015;91(4):320-5. doi: 10.1016/j.jped.2014.10.008. [PubMed: 25986614].

14. Sanchez-Roman FR, Perez-Lucio C, Juarez-Ruiz C, Velez-Zamora NM, Jimenez-Villarruel M. [Risk factors for asthenopia among computer terminal operators]. Salud Publica Mex. 1996;38(3):189-96. [PubMed: 8757544].

15. Han CC, Liu R, Liu RR, Zhu ZH, Yu RB, Ma L. Prevalence of asthenopia and its risk factors in Chinese college students. Int J Ophthalmol. 2013;6(5):718-22. doi: 10.3980/j.issn.2222-3959.2013.05.31. [PubMed: 24195055].

16. Yekta A, Fotouhi A, Hashemi H, Dehghani C, Ostadimoghaddam $\mathrm{H}$, Heravian J, et al. The prevalence of anisometropia, amblyopia and strabismus in schoolchildren of Shiraz, Iran. Strabismus. 2010;18(3):104-10. doi: 10.3109/09273972.2010.502957. [PubMed: 20843187].

17. Faghihi M, Ostadimoghaddam H, Yekta AA. Amblyopia and strabismus in Iranian schoolchildren, Mashhad. Strabismus. 2011;19(4):14752. doi: 10.3109/09273972.2011.622341. [PubMed: 22107119].

18. Razavi H, Kuper H, Rezvan F, Amelie K, Mahboobi-Pur H, Oladi MR, et al. Prevalence and causes of severe visual impairment and blindness among children in the lorestan province of iran, using the key informant method. Ophthalmic Epidemiol. 2010;17(2):95-102. doi: 10.3109/09286581003624954. [PubMed: 20302431].

19. Ma L, Ahati A, Liu XT, Zou ZY, Li Y, Wang MF, et al. [Epidemiology and related factor analysis of visual fatigue in university students of Haidian District of Beijing]. Beijing Da Xue Xue Bao. 2011;43(3):365-9. [PubMed: 21681265].

20. Ip JM, Robaei D, Rochtchina E, Mitchell P. Prevalence of eye disorders in young children with eyestrain complaints. Am J Oph- thalmol. 2006;142(3):495-7. doi: 10.1016/j.ajo.2006.03.047. [PubMed: 16935600].

21. Sterner B, Gellerstedt M, Sjostrom A. Accommodation and the relationship to subjective symptoms with near work for young school children. Ophthalmic Physiol Opt. 2006;26(2):148-55. doi: 10.1111/j.14751313.2006.00364.x. [PubMed: 16460315].

22. Mocci F, Serra A, Corrias GA. Psychological factors and visual fatigue in working with video display terminals. Occup Environ Med. 2001;58(4):267-71. doi: 10.1136/oem.58.4.267. [PubMed: 11245744].

23. Westman M, Liinamaa MJ. Relief of asthenopic symptoms with orthoptic exercises in convergence insufficiency is achieved in both adults and children. J Optom. 2012;5(2):62-7. doi 10.1016/j.optom.2012.03.002.

24. Owens DA, Wolf-Kelly K. Near work, visual fatigue, and variations of oculomotor tonus. Invest Ophthalmol Vis Sci. 1987;28(4):743-9. [PubMed: 3557879].

25. Mvitu MM, Kaimbo WK. [Manifestations of asthenopia in Black subjects]. Bull Soc Belge Ophtalmol. 2003(289):45-9. [PubMed: 14619629].

26. Neugebauer A, Fricke J, Russmann W. Asthenopia: frequency and objective findings. Ger J Ophthalmol. 1992;1(2):122-4. [PubMed: 1477627].

27. Alexander JA, Joyes D, Liew C, Omrod M, Wong A. A survey of cases in Sydney optometric practices: Age and sex distributions; symptoms and reasons for consultation. Aust J Optom. 1985;68(4):133-8.

28. McKay ME, Woodruff CE, Rumsey JM. Analysis of an optometry school's patient population. J Optom. 2002;27:381-5.

29. Scheiman M, Mitchell GL, Cotter S, Cooper J, Kulp M, Rouse M, et al. A randomized clinical trial of treatments for convergence insufficiency in children. Arch Ophthalmol. 2005;123(1):14-24. doi:10.1001/archopht.123.1.14. [PubMed: 15642806].

30. Scheiman M, Wick B. Clinical management of binocular vision: heterophoric, accommodative, and eye movement disorders. Lippincott Williams \& Wilkins; 2008.

31. Marran LF, De Land PN, Nguyen AL. Accommodative insufficiency is the primary source of symptoms in children diagnosed with convergence insufficiency. Optom Vis Sci. 2006;83(5):281-9. doi: 10.1097/01.opx.0000216097.78951.7b. [PubMed: 16699440]. 